
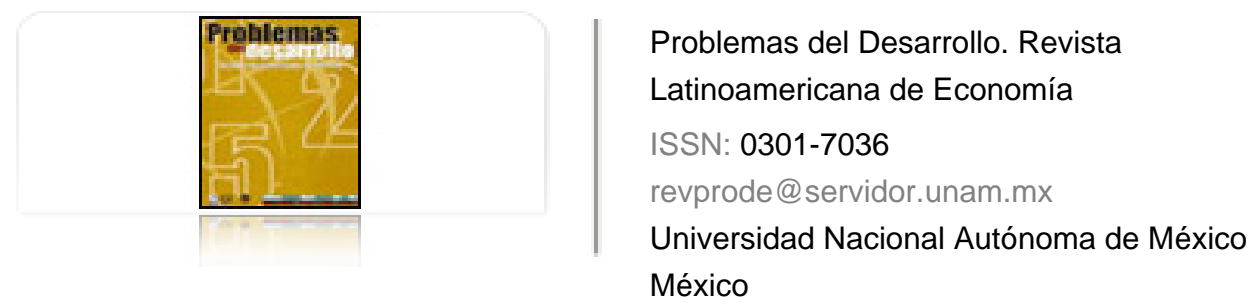

Reyes Guzmán, Gerardo

Incremento en los precios del maiz y la tortilla en México

Problemas del Desarrollo. Revista Latinoamericana de Economía, vol. 38, núm. 151, octubre-

diciembre, 2007, pp. 103-126

Universidad Nacional Autónoma de México

Distrito Federal, México

Disponible en: http://www.redalyc.org/articulo.oa?id=11820167006

- Cómo citar el artículo

- Número completo

- Más información del artículo

Página de la revista en redalyc.org

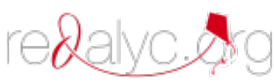

Sistema de Información Científica

Red de Revistas Científicas de América Latina, el Caribe, España y Portugal Proyecto académico sin fines de lucro, desarrollado bajo la iniciativa de acceso abierto 


\section{INCREMENTO EN LOS PRECIOS DEL MAÍZ Y LA TORTILLA EN MÉXICO}

\section{Gerardo Reyes Guzmán*}

Fecha de recepción: 20 de junio de 2007. Fecha de aceptación: 8 de octubre de 2007.

\section{Resumen}

George W. Bush propuso reducir en $20 \%$ el consumo de petróleo hasta 2017. Para ello se ha pensado tanto en etanol como en carbón líquido. Se planea incrementar la producción de etanol a 35 mil millones de galones al año y elevar los niveles de eficiencia en el uso de gasolina. Unos 35 mil millones de galones de etanol anuales para 2017 equivalen hoy a 317.5 millones de toneladas de maíz. Bajo las condiciones actuales es imposible alcanzar esa meta sin la ayuda de países latinoamericanos aliados a Estados Unidos. En consecuencia las economías involucradas enfrentan ya cuellos de botella que es necesario evaluar.

Palabras clave: maíz, etanol, biocomubustible, calentamiento atmosférico, tortilla, sector pecuario, inflación y pobreza.

* Profesor investigador de la Universidad Iberoamericana de Puebla. Correo electrónico: gerardo1960@yahoo.com. 


\section{Abstract}

George W. Bush proposed to reduce petroleum consumption 20\% by 2017. Both ethanol and liquid coal have been considered for this. It is planned to increase the production of ethanol to 35 billion gallons a year and to raise efficiency levels in the use of gasoline. An annual 35 billion gallons of ethanol in 2017 is equivalent today to 317.5 million tons of corn. Under present conditions it is impossible to achieve that target without the help of Latin American countries allied to the United States. As a consequence the economies involved are now facing a bottleneck that should be evaluated.

Key words: corn, ethanol, bio-fuel, atmospheric warming, tortilla, livestock sector, inflation and poverty.

\section{Résumé}

George W. Bush a proposé de réduire de $20 \%$ la consommation de pétrole d'ici à 2017. Pour cela, il a été pensé tant à l'éthanol qu'au charbon liquide. Il est prévu d'augmenter la production d'éthanol à 35 millions de gallons (132.3 millions de litres) par an et d'accroître l'efficience de l'usage de l'essence. 35 millions de gallons d'éthanol par an pour 2017 sont l'équivalent de 317,5 millions de tonnes de maïs. Dans les conditions actuelles, il est impossible d'atteindre ce but sans l'aide des pays latino-américains alliés aux États-Unis. En conséquence, les économies concernées se retrouvent d'ores et déjà face à des goulets d'étranglement qu'il est nécessaire d'évaluer.

Mots clés: maïs, éthanol, bio-combustible, réchauffement atmosphérique, tortilla, élevage, inflation et pauvreté.

\section{Resumo}

George W. Bush propôs reduzir em $20 \%$ o consumo de petróleo até 2017. Para isso pensou-se tanto em etanol como em carvão líquido. Planeja-se incrementar a produção de etanol até 35 bilhões de galões por ano e elevar os níveis de eficiência no uso de gasolina. Uns 35 bilhões de galões de etanol anuais para 2017 equivalem hoje a 317.5 milhões de toneladas de milho. Nas condições atuais é impossível alcançar essa meta sem a ajuda de países latino americanos aliados aos Estados Unidos. Em conseqüência as economias involucradas já enfrentam o efeito funil que é necessario avaliar.

Palavras chave: milho, etanol, biocomubustivel, aquecimento atmosférico, tortilla, setor pecuário, inflação e pobreza. 


\section{Introducción}

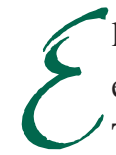

1 mercado energético ha presentado cambios estructurales que apuntan hacia el fin de los precios bajos del petróleo y gas natural. El precio del WTI (West Texas Intermediate) se incrementó casi cuatro veces al pasar de 19.68 dólares por barril en enero de 2002 a 76.85 dólares por barril en julio de 2006, oscilando entre 60 y 80 dólares por barril en lo que va de 2007. Si bien los precios actuales de estos hidrocarburos no reflejan escasez de manera fiel, pues éstos obedecen a movimientos geoestratégicos, el hecho es que han alcanzado niveles históricamente altos. Las políticas de producción de la Organización de Países Exportadores de Petróleo (OPEP) y el reacomodo de las potencias occidentales, así como el creciente peso, las llamadas economías BRIC (Brasil, Rusia, India y China) en los mercados internacionales de energía, constituyen un escenario complejo e incierto que dificulta estimar el comportamiento de los precios del petróleo y gas natural. En virtud de que la mayor parte de las reservas de petróleo y gas se encuentran en manos de gobiernos opuestos a los intereses de Estados Unidos o son zonas de conflicto, Arabia Saudita, Irán, Irak, Rusia, Venezuela, Bolivia, entre otros (Huntington, S., 1997; Brzezinski, Z., 1995; Brzezinski, Z., 1997), el gobierno estadounidense estudia seriamente poner un límite a su dependencia de hidrocarburos provenientes de esas zonas. Se trata del diseño de una estrategia de aseguramiento de energía para el futuro próximo, en un horizonte en que potencias como China, India y Rusia se perfilan como serios competidores en el mercado y finanzas internacionales. Estados Unidos, principal consumidor de crudo en el ámbito mundial, le apuesta al etanol como fuente de energía alternativa y como herramienta para desarticular el empoderamiento de los países exportadores de crudo, mediante la disminución futura de sus importaciones. El presente artículo examina las consecuencias que derivan de tal decisión, tanto en el escenario internacional como nacional. En el primer apartado se abunda sobre la importancia del uso de fuentes de energía más amigables para el ambiente, en aras de disuadir el peligro que representa el continuo calentamiento global provocado por la emisión de gases provenientes de fuentes de energía fósil, sobre todo petróleo y carbón. La segunda sección explora las implicaciones que se derivaron de un creciente uso de maíz amarillo para la elaboración de etanol en Estados Unidos. La tercera parte se ocupa de los efectos que tuvo el incremento de 
los precios internacionales del maíz en el mercado mexicano. El cuarto apartado evalúa el costo social y macroeconómico resultante de los ajustes en los precios de productos derivados del maíz. Finalmente, en las conclusiones se puntualiza el tipo de problemas que se generan a partir de este megaproyecto para América Latina en el futuro próximo.

\section{El cambio climático}

Estados Unidos (EU) es responsable de una quinta parte del total de emisiones de $\mathrm{CO}_{2}$ en el mundo. El consumo de petróleo per capita asciende a siete mil 835 litros, mientras que, por ejemplo, países como India requieren 439 litros por persona al año (Sen, 2007). En su informe de gobierno (23 de enero de 2007), el presidente George W. Bush decide reivindicar su fracaso en la guerra de Irak apelando a una nueva amenaza para la sociedad estadounidense: el peligro que representa el cambio climático y la urgencia para detenerlo. A partir de la hecatombe que dejó atrás el huracán Katrina en septiembre de 2005, la ola de calor que atacó la costa oeste en 2006, el derretimiento de los glaciares y el repentino deceso de osos polares, el cambio climático se ha convertido en prioridad para la Casa Blanca y es bandera electoral de los candidatos a la presidencia en 2008. Inclusive el sector privado, renuente antaño a toda iniciativa por controlar las emisiones de $\mathrm{CO}_{2}$, ha comenzado a ver oportunidades de negocio en este nuevo reto. Análogamente, grupos de derecha radicales conocidos como los halcones se suman al proyecto en aras de reducir la dependencia que EU tiene del petróleo procedente de regiones hostiles a los intereses de Washington, por ejemplo Venezuela y Medio Oriente. Por su parte, los productores agrícolas, receptores de generosos subsidios, apoyan con entusiasmo la iniciativa. Estados Unidos otorga ya subsidios al etanol por 17 mil 190 millones de dólares.

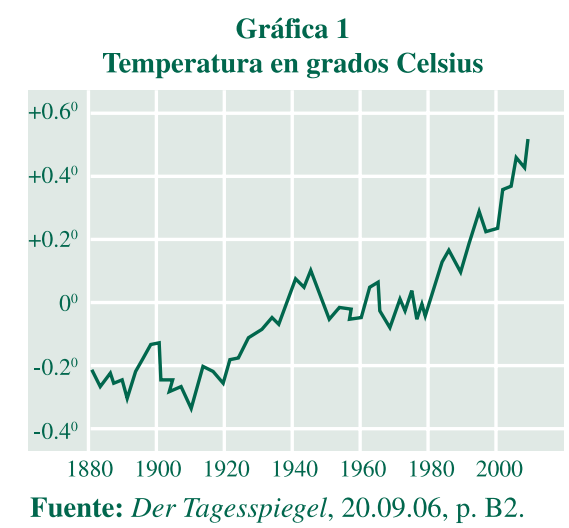


Los candidatos a la presidencia John McCain, Hillary Clinton y Barack Obama proponen la puesta en marcha del sistema de bonos que permiten a las empresas emitir un cierto volumen de contaminantes, lo que redunda en la comercialización de aquellos que las compañías no utilizan en la medida en que se vuelvan más limpias (The Economist, 27 de enero de 2007:9).

George W. Bush propuso reducir en 20\% el consumo de petróleo para 2017. Para ello se ha pensado en etanol, carbón líquido, etanol de celulosa y biodiesel. Se planea incrementar la producción de etanol a 35 mil millones de galones al año y elevar los niveles de eficiencia en el uso de gasolina. Desde la toma del Congreso por los demócratas en las elecciones medias de noviembre de 2006, el número de iniciativas de ley orientadas a revertir el calentamiento atmosférico ha sido inusitado. Se intenta cobrar impuestos por la producción de crudo y penalizar a las empresas que se nieguen a renegociar los permisos que el gobierno otorgó en el pasado para contaminar sin asumir costo alguno. Se espera recaudar un monto de 15 mil millones de dólares que se utilizarán para financiar proyectos de energía renovable.

Según el semanario inglés The Economist (9 de septiembre de 2006), el clima casi no ha cambiado desde la revolución industrial. Durante el siglo XIX la temperatura fue estable; subió durante la primera mitad del siglo XX; descendió entre los años 1950 y 1970 , y desde ese periodo a la fecha comenzó a incrementarse, especialmente a partir de la década de los ochenta hasta alcanzar los $0.6^{0} \mathrm{C}$ en fechas recientes (véase la gráfica 1). Se afirma que en 1975 la revista Newsweek publicó un artículo titulado The Cooling World, en donde se hacía hincapié en la drástica disminución en la producción de alimentos a partir del enfriamiento atmosférico. Se pensaba que el sulfuro y otras partículas en el aire hacían que los rayos del sol se reflejaran antes de que pudieran calentar la atmósfera. Para finales del siglo XX los esfuerzos por controlar ese tipo de sustancias tuvieron éxito y la atmósfera se comenzó a calentar. A partir de ese momento, la preocupación sobre el calentamiento atmosférico ha estado cobrando cada vez más importancia. Los datos suelen contradecirse a corto plazo, en tanto que las predicciones de largo plazo son inexactas. Áreas en donde los glaciares se contraen pueden ser compensadas por otras en donde aumentan; lo mismo sucede con los niveles de precipitación pluvial. No obstante, se ha desencadenado toda una ola de investigaciones y preocupaciones acerca del cambio climático por parte de gobiernos, empresas y universidades. Inclusive la Organización de las Naciones Unidas (ONU) ha convocado a un grupo de especialistas para desarrollar la UNEP (United Nations Environmental Program), y en conjunto con la Organización 
Mundial de Meteorología informan sobre el cambio climático por medio del IPPC (Intergovernmental Panel on Climate Change). En 2001, el reporte del IPPC señaló que la temperatura podría incrementarse entre $1.4^{0} \mathrm{C}$ y $5.8^{\circ} \mathrm{C}$ a finales de este siglo. Por otro lado, observaciones satelitales confirman el calentamiento atmosférico. Se puntualiza que el hielo del mar ártico se derrite en $9 \%$ cada 10 años. Análogamente, la correlación entre el calentamiento atmosférico y la intensidad de los huracanes se ha vuelto más estrecha. Se estima que el nivel del mar puede elevarse a partir de la expansión térmica en 60 centímetros para 2100. Severas sequías se prevén en África Subsahariana, el Amazonas y Australia, así como la desaparición de miles de especies.

En 1997 se reunieron los países miembros de la ONU para firmar el protocolo de Kioto, como un compromiso para disminuir las emisiones de gases causantes del efecto invernadero en 5.2\% de 2008 a 2012, teniendo como referencia los niveles de contaminantes registrados en 1990. Se trata de los siguientes gases: dióxido de carbono $\left(\mathrm{CO}_{2}\right)$, metano $\left(\mathrm{CH}_{4}\right)$ y óxido nitroso $\left(\mathrm{N}_{2} \mathrm{O}\right)$, además de tres gases industriales fluorados: hidrofluorocarbonos (HFC), perfluorocarbonos (PFC) y hexafluoruro de azufre $\left(\mathrm{SF}_{6}\right)$. Este $5.2 \%$ es en el ámbito global, lo que implica que cada país establece sus propios porcentajes. Se acordó que el compromiso sería obligatorio cuando lo avalaran los países industrializados, responsables de 55\% del total de $\mathrm{CO}_{2}$ en la atmósfera. Con la ratificación de Rusia en noviembre de 2004, el protocolo de Kioto entró en vigor el 16 de febrero de 2005. Asimismo, se promovió la generación de un desarrollo sostenible mediante el uso de energías alternativas como la solar. Estados Unidos firmó el protocolo pero no lo ratificó, al aducir que si no se lograba involucrar a China e India, la economía estadounidense se vería injustamente desfavorecida. Otros países que se negaron a convalidar el protocolo fueron Australia, Mónaco y Lichtenstein. En la Unión Europea, el mejor desempeño lo ha tenido Gran Bretaña, nación cuya economía creció en 36\% de 1990 a 2002, mientras que sus emisiones se redujeron en $15 \%$. En contraste, España incrementó sus emisiones en $45.61 \%$ de 1990 a 2004, lo cual rebasa el límite permitido en el protocolo de Kioto que es de $15 \%$ durante dicho lapso.

\section{Estados Unidos, primer productor mundial de maíz}

De 1960 a 2000 la producción mundial de maíz se incrementó en 28\% cada 10 años. A partir de 2000 las tasa anual de crecimiento fue de $2.6 \%$, alcanzando un volumen de 692.7 millones de toneladas (mt) en 2006 (véase grafica 2). Si la tendencia sigue, para 2010 la producción mundial de maíz sería de 744 mt. 
Gráfica 2

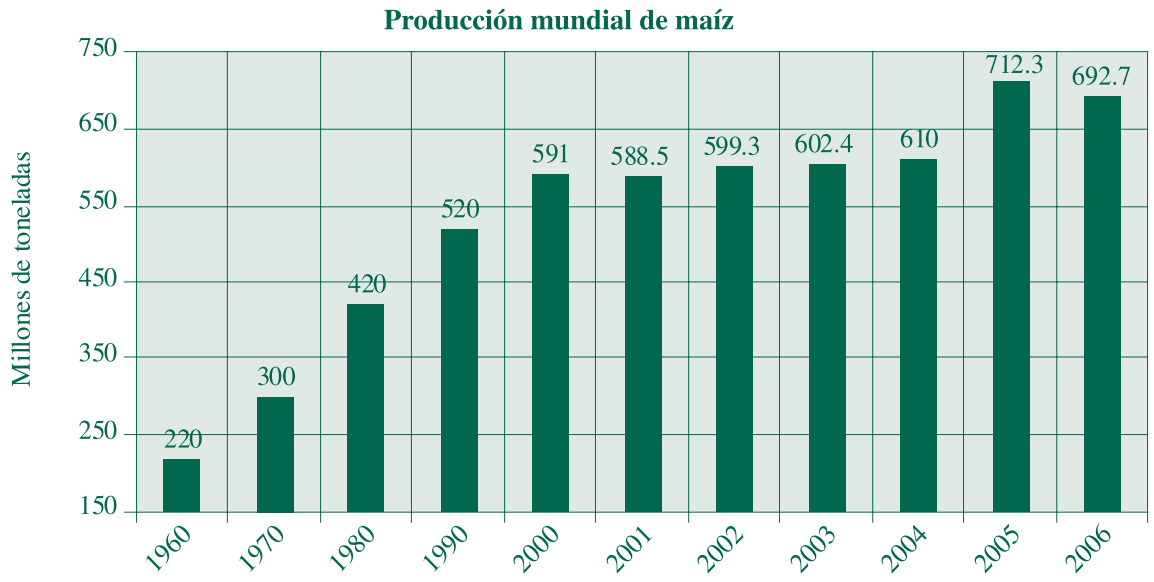

Fuente: Grefe, Christiane, "Mais für die Welt", Die Zeit, 19 de agosto 2004, pp. 11-14. National Corn Growers Asociation, World of corn production, 2007, [http://www.ncga.com/WorldOfCorn/main/ production1.asp]

Como se puede observar en el cuadro 1, Estados Unidos mantienen el liderazgo entre los primeros productores mundiales de maíz, seguidos por China. Por otro lado, mientras la Unión Europea y Brasil se fortalecen, México y Argentina pierden participación pasando de $3.17 \%$ y $2.83 \%$, respectivamente, en el periodo $1999-2000$, a $2.81 \%$ y $2.09 \%$ en $2005-2006$, lo que indica que la producción mundial tiende a concentrarse entre los cuatro primeros.

Cuadro 1

Participación de los seis grandes productores en el total

\begin{tabular}{lcccccc}
\hline & $99 / 00$ & $00 / 01$ & $01 / 02$ & $02 / 03$ & $04 / 05$ & $05 / 06$ \\
\multicolumn{1}{c}{ País } & $\%$ & $\%$ & $\%$ & $\%$ & $\%$ & $\%$ \\
\hline EU & 39.44 & 42.79 & 40.29 & 37.98 & 42.1 & 40.75 \\
China & 21.09 & 18.01 & 19.04 & 20.13 & 18.29 & 20.12 \\
UE & 5.99 & 6.43 & 6.67 & 7.47 & 7.51 & 6.97 \\
Brasil & 5.21 & 7.06 & 5.92 & 6.65 & 4.91 & 5.92 \\
México & 3.17 & 3.04 & 3.40 & 3.12 & 3.1 & 2.81 \\
Argentina & 2.83 & 2.62 & 2.45 & 2.57 & 2.88 & 2.09 \\
Total & $\mathbf{7 7 . 7 3}$ & $\mathbf{7 9 . 9 5}$ & $\mathbf{7 7 . 7 7}$ & $\mathbf{7 7 . 9 2}$ & $\mathbf{7 8 . 7 9}$ & $\mathbf{7 8 . 6 6}$ \\
\hline
\end{tabular}

Fuente: Elaboración propia con datos de National Corn Growers Asociation, 2007, World of corn production: [http://www.ncga.com/WorldOfCorn/main/production1.asp].

Por otro lado, la participación de EU en el consumo mundial de la gramínea se incrementó de $31.78 \%$ en el periodo $1999-2000$ a $33.09 \%$ en $2005-2006$, mientras que China lo hizo de $19.48 \%$ a $19.57 \%$. Los demás consumidores mantuvieron casi constante su participación a lo largo del periodo como lo muestra el cuadro 2. 
Cuadro 2

Participación de los cuatro principales consumidores mundiales de maíz en la demanda total

\begin{tabular}{lrrrrrrr}
\hline & $99 / 00$ & $00 / 01$ & $01 / 02$ & $02 / 03$ & $03 / 04$ & $04 / 05$ & $05 / 06$ \\
\hline EU & 31.78 & 32.57 & 32.81 & 31.94 & 32.52 & 32.79 & 33.09 \\
China & 19.48 & 19.72 & 20.12 & 20.03 & 20.28 & 19.12 & 19.57 \\
Brasil & 5.53 & 5.67 & 5.63 & 5.78 & 5.81 & 5.62 & 5.57 \\
Mexico & 3.91 & 3.95 & 3.85 & 3.91 & 4.04 & 4.07 & 3.9 \\
\hline
\end{tabular}

Fuente: Elaboración propia con datos de National Corn Growers Asociation (2007). World of corn production: [http://www.ncga.com/WorldOfCorn/main/production1.asp].

En la gráfica 3 se aprecia la producción anual de maíz en Estados Unidos, que se incrementó de $161 \mathrm{mt}$ en 1993 a $267 \mathrm{mt}$ en 2006, lo que implica un crecimiento anual de $3.96 \%$. Se puede observar que EU tiene una influencia importante sobre los precios internacionales del maíz. Por ejemplo, en 1995, cuando la producción cayó a 187.97 $\mathrm{mt}$, el precio por bushel fue de 3.24 dólares; posteriormente, conforme se robustece la producción el precio cae hasta alcanzar su nivel más bajo de 1.82 dólares por bushel en 1999. Resalta la disminución de la producción de 300 mt en 2004 a 267.6 mt en 2006, lo que se tradujo en un repunte del precio de 2.06 a 3.2 dólares por bushel. Análogamente, los inventarios finales se redujeron 64\% al pasar de $53.7 \mathrm{mt}$ en 2004-2005 a $19.10 \mathrm{mt}$ en 2006-2007. Para marzo de 2007, el precio del maíz se ubicaba arriba de los cuatro dólares por bushel en el mercado de futuros.

Gráfica 3

Producción en EU y precio internacional del maíz

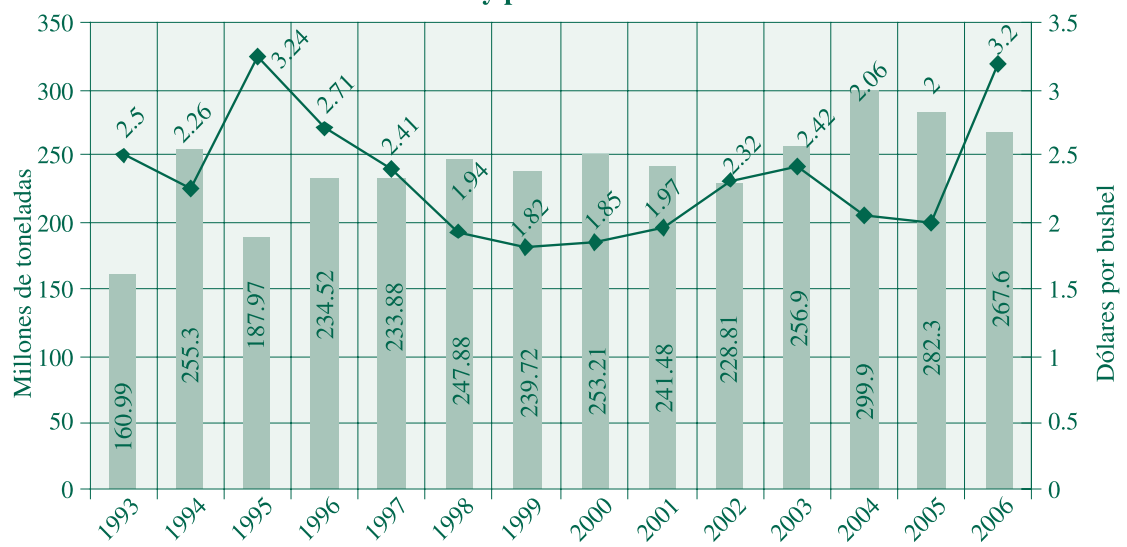

Fuente: Elaboración propia con datos de National Corn Growers Asociation (2007). World of corn production: [http://www.ncga.com/WorldOfCorn/main/production1.asp].

La gráfica 4 muestra la relación entre las exportaciones de Estados Unidos y el precio internacional del maíz. Las cifras indican que las exportaciones responden de 
manera dinámica ante un aumento de los precios. Así, por ejemplo, éstas alcanzan su nivel más alto en 1995, año en que la producción fue sólo de $187.7 \mathrm{mt}$, pero el precio es de 3.24 dólares por bushel. En general, las exportaciones no volvieron a rebasar las 51 mt hasta 2006, año en que, motivadas por un incremento en los precios, ascendieron a $56.2 \mathrm{mt}$. El aumento de las exportaciones ante una caída de la producción se posibilita con el uso de las reservas para satisfacer la demanda. Ello se reflejó en repuntes importantes en el valor de las exportaciones que fueron de 24.12 mil millones de dólares en 1995 y de 33.7 mil millones de dólares en 2006, cifra récord en el rubro.

Gráfica 4

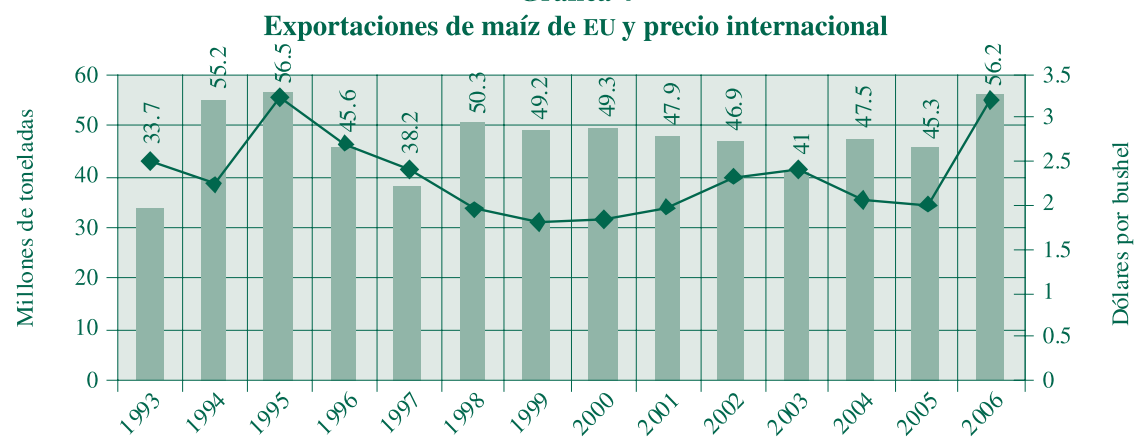

Fuente: Elaboración propia con datos de National Corn Growers Asociation, 2007, World of corn production: [http://www.ncga.com/WorldOfCorn/main/production1.asp].

Por otro lado, más de 65\% de la producción en EU se concentra en Iowa, Illinois, Minesota, Nebraska e Indiana, lo que explica la dependencia del precio internacional del maíz en el desempeño productivo de esos estados, miembros a su vez del llamado "cinturón del maíz".

El aumento inusitado en el precio del maíz entre finales de 2006 y principios de 2007 se atribuye, entre otras cosas, ${ }^{1}$ a la producción de etanol. La generación mundial de este energético se elevó en $20 \%$ de 2000 a 2005. Se calcula que Eu y Brasil

1 En general se mencionan factores como: el aumento de la demanda mundial de granos, los altos precios de petróleo, los huracanes, y los llamados biocomubustibles. Pero también es un problema de paridad cambiaria. Según Meter Timmer, economista agrónomo del Center for Global Development, el dólar se ha depreciado con respecto a la mayoría de las divisas internacionales fuertes. Debido a que los granos se comercian en dólares, las presiones infla cionarias en EU derivan en un dólar débil. En euros, el incremento de los granos no ha sido sustancial. El mal tiempo ha jugado un papel importante; por ejemplo, en Australia la sequía hizo descender las exportaciones de trigo en 20\%. En China, las inundaciones arrasaron con 5.5 millones de hectáreas de trigo y semillas maduras. Los altos precios del crudo se mani fiestan también en un encarecimiento de petroquímicos como es le caso de los materiales de empaque y embalaje y el polipropileno, cuyo precio se incrementó en 70\% de 2003 a 2007 (Sheridan, A., 2007:34.). 
aportan $70 \%$ de la producción mundial casi en partes iguales; el primero con maíz y el segundo con caña de azúcar. El etanol se utiliza en la elaboración de combustible combinado con gasolina, lo cual incrementa el octanaje y reduce la emisión de gases. Desde la década de los setenta, Brasil ha producido etanol y ha logrado importantes ahorros de divisas en momentos en que los precios del petróleo han sido altos (Nitsch y Giersdorf, 2005). Estados Unidos ha logrado reducir los costos de producción de 1.4 dólares por galón en 1980 a 1.10 dólares a inicios de la presente década. En el cuadro 3 se puede ver que los cinco estados mencionados poseen 62 de las 109 plantas que existían en 2006 en EU. Según información del Departamento de Agricultura estadounidense y ASERCA, 42 plantas están en fase de construcción y siete en proceso de expansión. La producción total de etanol en 2006 fue de cinco mil 836 millones de galones, de los cuales $68.8 \%$ fue producido por los estados que aparecen en el cuadro 3. En este contexto, Iowa produjo, con sus 26 plantas, mil 698.5 millones de galones equivalentes a $31.53 \%$ de toda la producción.

Cuadro 3

Estados productores de maíz y etanol en EU

\begin{tabular}{lcccc}
\hline & $\begin{array}{c}\text { \% Producción total } \\
\text { de maíz 2002 }\end{array}$ & $\begin{array}{c}\text { \% Producción total } \\
\text { de maíz 2006 }\end{array}$ & $\begin{array}{c}\text { Plantas de etanol } \\
\text { \% Producción total } \\
\text { de etanol }\end{array}$ \\
\hline Iowa & 21.8 & 19.46 & 26 & 31.53 \\
Illinois & 16.6 & 17.24 & 6 & 13.05 \\
Minesota & 11.6 & 10.46 & 17 & 10.04 \\
Nebraska & 10.4 & 11.18 & 12 & 12.17 \\
Indiana & 7 & 8.01 & 1 & 1.89 \\
Total & $\mathbf{6 7 . 4}$ & $\mathbf{6 6 . 3 5}$ & $\mathbf{6 2}$ & $\mathbf{6 8 . 6 8}$ \\
\hline
\end{tabular}

Fuente: Elaboración propia con datos de National Corn Growers Asociation (2007). World of corn production: [http://www.ncga.com/WorldOfCorn/main/production1.asp].

En el cuadro 4 se puede advertir que el volumen de maíz destinado para la producción de etanol pasó de 10.9 millones de toneladas - equivalentes a $4.6 \%$ de la producción total y a 23.6\% de las exportaciones — en 1996 a 54.6 millones de toneladas - equivalentes a $24.4 \%$ de la producción total y $97.1 \%$ de las exportacionesen 2006. Probablemente para 2007 el volumen de maíz para etanol supere el de las exportaciones. Este incremento en 10 años equivale a un crecimiento anual promedio de $17.48 \%$, con lo que, según los datos de 2006, se espera que la producción en 2017 sea de 12 mil 648.58 millones de bushel o 321.27 millones de toneladas. Esta cifra rebasa cualquier referente histórico en EU, pues al retomar el volumen de producción total de maíz en 2006 — que fue de 267 mt—, así como la tasa de crecimiento del mismo calculada en 3.96\% anual, se tiene que para 2017 la producción total en Estados Unidos sería de 410.21 millones de toneladas, lo que implica que bajo las 
Cuadro 4

Maíz destinado a la producción de etanol en los Estados Unidos (Diferentes equivalencias)

\begin{tabular}{ccccc}
\hline & $\begin{array}{c}\text { Millones } \\
\text { de galones }\end{array}$ & $\begin{array}{c}\text { Millones } \\
\text { de litros }\end{array}$ & $\begin{array}{c}\text { Millones } \\
\text { de Bushel }\end{array}$ & $\begin{array}{c}\text { Millones } \\
\text { de toneladas }\end{array}$ \\
\hline 1996 & $1,201.2$ & $4,546.8$ & 429 & 10.90 \\
1997 & $1,346.8$ & $5,097.9$ & 481 & 12.22 \\
1998 & $1,472.8$ & $5,574.8$ & 526 & 13.36 \\
1999 & $1,584.8$ & $5,998.7$ & 566 & 14.38 \\
2000 & $1,758.4$ & $6,655.9$ & 628 & 15.95 \\
2001 & $1,976.8$ & $7,482.6$ & 706 & 17.93 \\
2002 & $2,788.8$ & $10,556.1$ & 996 & 25.30 \\
2003 & $3,256.4$ & $12,326.1$ & 1,163 & 29.54 \\
2004 & $3,872.4$ & $14,657.8$ & 1,383 & 35.13 \\
2005 & $4,485.6$ & $16,978.9$ & 1,602 & 40.69 \\
2006 & $6,020.0$ & $22,786.9$ & 2,150 & 54.61 \\
\hline
\end{tabular}

Fuente: National Corn Growers Asociation, World of corn production, 2007: [http://www.ncga.com /WorldOfCorn/main/production1.asp].

condiciones actuales el volumen destinado a la producción de etanol equivaldría a $78.31 \%$ de la producción total. Ello implicaría que otros consumidores tradicionales, especialmente las dinámicas industrias pecuaria y almidonera, estarían enfrentando una grave crisis de escasez del grano (véase la gráfica 5).

Gráfica 5

Producción de maíz en los Estados Unidos (Proyección 2006-2017)

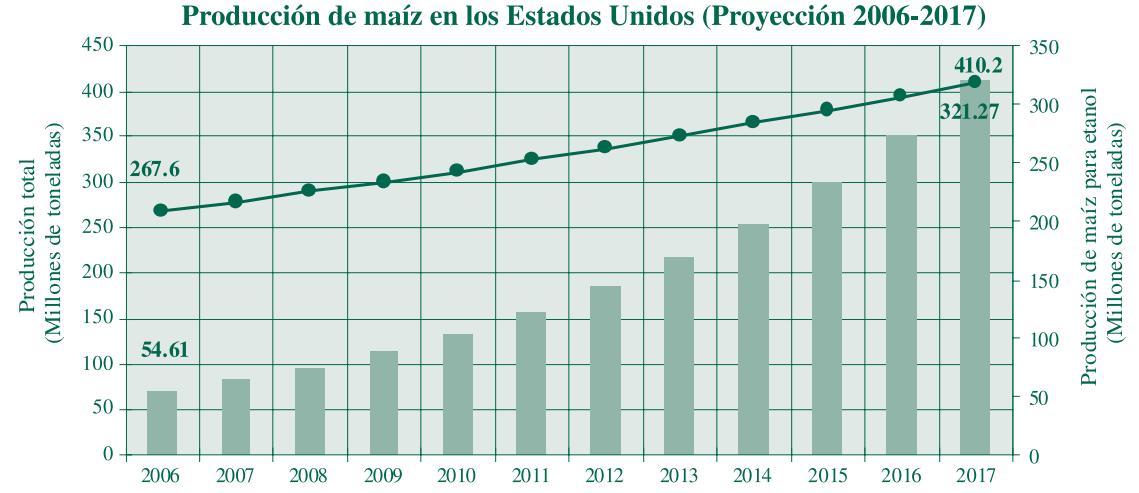

Fuente: Elaboración propia con datos de National Corn Growers Asociation, 2007, World of corn production: [http://www.ncga.com/WorldOfCorn/main/production1.asp].

Como se mencionó en el primer apartado, Estados Unidos se ha propuesto alcanzar una capacidad productiva de 35 mil millones de galones de etanol anuales para 2017. Si un bushel produce 2.8 galones de etanol entonces se habla de 12 mil 500 millones de bushel o $317.5 \mathrm{mt}$, cifra ligeramente inferior a nuestras proyecciones (321.27 mt). El reto es cómo aumentar la producción para satisfacer esa demanda, 
pues tan sólo para conservar $24.4 \%$ de participación de maíz para etanol que se registró en 2006, la producción para 2017 tendría que ser de mil 301.2 mt y no de 410.2 como arrojan las estimaciones.

La visita del presidente George W. Bush a América Latina durante el primer trimestre de 2007 tuvo el propósito de asegurar el suministro de etanol para lograr la meta. En la actualidad, el etanol está protegido en Estados Unidos con un arancel de $54 \%$ en beneficio de los lobbies agropecuarios. No obstante la imposibilidad de responder a las necesidades que pueda requerir el consumo nacional, se piensa abrir el mercado. El 8 de marzo de 2007, George Bush y el primer mandatario brasileño Luiz Inacio Lula da Silva anunciaron su intención de sumar esfuerzos para promover la producción de etanol. Brasil destaca por su éxito y experiencia en la generación del biocombustible. Según datos publicados por The Economist (3 de marzo de 2007: $44)$, ese país sudamericano produce un litro de etanol a un costo de 22 centavos de dólar por litro, mientras que en Estados Unidos cuesta 30 centavos. Ello se debe a que una etapa previa para producir etanol es obtener edulcorante, paso que Brasil se ahorra con caña de azúcar. Durante las tres últimas décadas, las plantaciones de caña de azúcar se han expandido en el norte y oeste del país, reemplazando otros cultivos como café, cítricos y pastizales. Se planean inversiones de 12.2 mil millones de dólares (mmd) para la construcción de 77 plantas de etanol dentro de los próximos cinco años, así como 2.4 mmd para expandir la existentes. Para 2012, un total de 412 destilerías estarían produciendo 9.5 mil millones de galones. Si Estados Unidos liberaliza el mercado de etanol, Brasil podría elevar sus exportaciones de tres mil millones de litros (793 millones de galones) en la actualidad a 200 mil millones de litros (52.8 mil millones de galones) para 2025, cifra que podría sustituir una décima parte del consumo mundial actual de gasolina.

Por su parte, Colombia también se suma al proyecto de George W. Bush. Posee cinco destilerías que producen 360 millones de litros de etanol al año utilizando caña de azúcar. El gobierno instituyó una ley que obliga a la mezcla de etanol con gasolina, con participación creciente del primero. Para 2009 se espera que sea de $10 \%$ hasta llegar a un máximo de $25 \%$. Costa Rica y Panamá van por el mismo camino, lo que revela que este mercado favorecerá a productores de Centroamérica, el Caribe y la región andina. La firma estadounidense Maple planea invertir 120 millones de dólares en una planta de etanol en Perú, de donde se espera crear tres mil 200 empleos. Esta planta podría abastecer a Estados Unidos por medio de un ducto que la comunique con el océano Pacífico. Se esperan exportar 120 millones de litros año para 2010 hasta alcanzar una capacidad exportadora de 400 millones de litros anuales. En México, 
empresas como Destilmex, Mexstarch, Cargill y Jalcohol ya están involucradas en la construcción de las primeras plantas de etanol en Sinaloa, Veracruz y Chihuahua, con una inversión de 88 millones de dólares. El Banco Interamericano de Desarrollo argumenta que reemplazar $10 \%$ del consumo de gasolina con etanol refinado produciría a México un ahorro de dos mmd al año y crearía 400 mil empleos (The Economist, 3

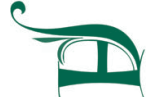
de marzo de 2007:46).

Un actor decisivo en la demanda de etanol será la industria automotriz, ya que se ha propuesto reducir la cantidad de $\mathrm{CO}_{2}$ en gramos por kilómetro de 161.7 en la actualidad a sólo 120 en el futuro. Ello requerirá no sólo de los motores híbridos que funcionan con energía eléctrica y gasolina, sino de la utilización creciente del etanol. Por ejemplo, se afirma que un vehículo Chevrotlet 2006 da un rendimiento con gasolina de 21 y 31 millas por galón (mpg) en ciudad y carretera respectivamente; no obstante, cuando se utiliza la mezcla E85 (85\% etanol y 15\% gasolina) que se comercializa en algunas ciudades de Estados Unidos, el rendimiento se reduce a 16 y $23 \mathrm{mpg}$ respectivamente. Existe también la mezcla E10 con sólo 10\% de etanol y que incrementa sustancialmente el rendimiento de los vehículos, lo que hace pensar que será el mercado por medio de los precios el que determine la demanda efectiva de etanol en comparación con la gasolina (Dudenhöffer, 22 de febrero de 2007:22).

\section{El mercado del maíz en México}

El cuadro 5 muestra el comportamiento del mercado nacional del maíz. La producción nacional creció de 18 mt en 1996 a 21.2 mt en 2006, equivalente a un incremento anual de $1.64 \%$. La producción es predominantemente de maíz blanco y México es el país que aporta 50\% de la oferta mundial de este tipo de gramínea. Por otro lado, la

Cuadro 5

Oferta y demanda de maíz en México

(Millones de toneladas)

\begin{tabular}{cccc}
\hline Año & Producción & Importaciones & Demanda \\
\hline 1996 & 18.0 & 5.89 & 23.9 \\
1997 & 17.7 & 2.53 & 20.2 \\
1998 & 18.5 & 5.281 & 23.7 \\
1999 & 17.7 & 5.6 & 23.3 \\
2000 & 17.6 & 5.55 & 23.1 \\
2001 & 20.1 & 7 & 27.1 \\
2002 & 19.3 & 7.45 & 26.7 \\
2003 & 20.7 & 8.4 & 29.1 \\
2004 & 21.7 & 7.74 & 29.4 \\
2005 & 22.7 & 8.43 & 31.1 \\
2006 & 21.2 & 10.73 & 31.9 \\
\hline
\end{tabular}

Fuente: El Financiero, 26 de enero de 2007, p. 19). 
demanda aumentó de $23.9 \mathrm{mt}$ a $31.9 \mathrm{mt}$ en el mismo periodo, lo que se traduce en un incremento anual de $2.9 \%$. La diferencia resultante explica el crecimiento inusitado de las importaciones que pasaron de $5.9 \mathrm{mt} \mathrm{a} 10.73 \mathrm{mt}$, es decir, más de $6 \%$ anual. Por su parte, la demanda se compone de maíz blanco y maíz amarillo, como lo muestra la cuadro 6. El consumo de maíz blanco es relativamente estable y se mantiene en los $10.5 \mathrm{mt}$ anuales. La industria de la masa y la tortilla requiere de tres $\mathrm{mt}$ al año, tres millones consumen la harinera y tres millones la de tradición rural, destinándose el resto al consumo animal. Llama la atención, sin embargo, el consumo de maíz amarillo, pues de 2001 a 2004 creció de 12.6 mt a 17.9 mt anuales. Este despunte se debe principalmente al dinamismo de la industria pecuaria que pasó de $10 \mathrm{mt}$ a $15.3 \mathrm{mt}$ en el periodo en cuestión, lo cual equivale a un crecimiento anual de $11.21 \%$. Análogamente, la participación de este sector en la demanda total de maíz amarillo creció de $43.3 \%$ a $53.8 \%$ en el periodo que nos ocupa.

Por otro lado y a pesar de que la industria almidonera haya registrado un consumo estable, la importancia de la fructosa a base de maíz en el mercado de edulcorantes sugiere que en el futuro es probable que aumente su consumo. Del cuadro 6 también se desprende que no hay escasez de maíz blanco, puesto que su demanda es muy inferior a la oferta principalmente de origen nacional (véase cuadro 5). El problema se centra en el abastecimiento de maíz amarillo, cuya demanda de la industria pecuaria y almidonera es creciente y no se tiene capacidad interna para satisfacerla, aun echando mano de maíz blanco. Si a ello se agregan los planes de expansión de la generación de etanol, los productores mexicanos se verán rebasados por el volumen requerido en los próximos años, recurriendo a las importaciones y a un fuerte impulso de la producción nacional vía aplicación de nuevas tecnologías y expansión de la superficie cosechada. Por ejemplo y según el especialista Antonio Turrent, existen condiciones de suelo, clima y agua para elevar la producción maicera. Afirma que hay dos millones de hectáreas dispersas en ocho estados del sur-sureste del país que permanecen ociosas en el ciclo otoño-invierno.

El incremento de los precios internos del maíz es un reflejo de los precios internacionales determinados principalmente por Estados Unidos. Éstos no reflejan una escasez del maíz blanco en México, pero ofrecen a los intermediarios una atractiva forma de especular. En menos de ocho meses el precio promedio por tonelada en México aumentó de mil 750 a tres mil 500 pesos a raíz de una escasez manipulada por grandes empresas comercializadoras, así como de prácticas monopólicas en la cadena productiva maíz-tortilla. Con el permiso para importar libre de aranceles que extendió el gobierno federal a principio de 2007 en aras de combatir el desabasto, 
se benefició a un reducido y poderoso grupo de empresas con extensa capacidad logística y de almacenamiento.

\section{Cuadro 6}

Demanda estimada nacional de maíz: 2001-2006

(Millones de toneladas y participacion de sectores)

\begin{tabular}{|c|c|c|c|c|c|c|c|c|c|c|c|c|}
\hline Concepto & 2001 & $\%$ & 2002 & $\%$ & 2003 & $\%$ & 2004 & $\%$ & $2005^{*}$ & $\%$ & $2006 *$ & $\%$ \\
\hline $\begin{array}{l}\text { Industria } \\
\text { de la harina }\end{array}$ & 3 & 12.9 & 3.4 & 13.7 & 3.6 & 13.7 & 3 & 10.5 & 3 & 10.1 & 3 & 9.5 \\
\hline $\begin{array}{l}\text { Industria de } \\
\text { la masa } \\
\text { y la tortilla }\end{array}$ & 2.8 & 12.1 & 3.3 & 13.3 & 3.5 & 13.3 & 2.8 & 9.85 & 3 & 10.1 & 3 & 9.5 \\
\hline $\begin{array}{l}\text { Tortilla de } \\
\text { tradición rural }\end{array}$ & 2.9 & 12.5 & 2.9 & 11.7 & 2.9 & 11 & 2.9 & 10.2 & 2.5 & 8.4 & 2.5 & 7.9 \\
\hline $\begin{array}{l}\text { Consumo } \\
\text { animal } \\
\text { (sector rural) }\end{array}$ & 1.8 & 7.7 & nd & - & nd & - & 1.8 & 6.3 & 1.3 & 4.4 & 1.3 & 4.1 \\
\hline $\begin{array}{l}\text { Total maíz } \\
\text { maíz blanco }\end{array}$ & 10.5 & 45.4 & 9.6 & 38.7 & 10 & 38.1 & 10.5 & 36.9 & 9.8 & 32.9 & 9.8 & 31.0 \\
\hline $\begin{array}{l}\text { Industria } \\
\text { almidonera }\end{array}$ & 2.2 & 9.5 & 2.5 & 10 & 3 & 11.4 & 2.2 & 7.7 & 2.2 & 7.4 & 2.2 & 7.0 \\
\hline $\begin{array}{l}\text { Cereales } \\
\text { y botanas }\end{array}$ & 0.4 & 1.7 & 0.4 & 1.6 & 0.6 & 2.3 & 0.4 & 1.4 & 1.75 & 5.9 & 1.75 & 5.5 \\
\hline $\begin{array}{l}\text { Consumo } \\
\text { total } \\
\text { pecuario** }\end{array}$ & 10 & 43.29 & 12.3 & 49.6 & 12.6 & 48 & 15.3 & 53.8 & 16 & 53.8 & 17.9 & 56.6 \\
\hline $\begin{array}{l}\text { Total maíz } \\
\text { amarillo }\end{array}$ & 12.6 & 54.5 & 15.2 & 61.2 & 16.2 & 61.8 & 17.9 & 63 & 19.95 & 67.1 & 21.85 & 69.1 \\
\hline $\begin{array}{l}\text { Demanda } \\
\text { total de maíz }\end{array}$ & 23.1 & - & 24.8 & - & 26.2 & - & 28.4 & - & 29.8 & & 31.7 & \\
\hline $\begin{array}{l}\text { Variación } \\
\text { porcentual } \\
100 \%=2000\end{array}$ & 112.6 & & 120.9 & & 127.8 & & 138.5 & & 1.45 & & 1.54 & \\
\hline
\end{tabular}

* Estimación propia con base en tendencias y cifras de 2001-2004. El Financiero (30 de abril de 2007:57).

** Incluye importaciones de maíz quebrado, sorgo equivalente a maíz e importaciones no controladas. Fuente: Elaboración propia con datos de la Cámara Nacional de Maíz Industrializado; Luis Ángel López Ibarra (2003). Perspectivas del maíz para 2003, México, Banco de México-FIRA; El Financiero (20 de Julio de 2004). Promueven industriales aumento en la producción de maíz, p. 30.

Un problema adicional lo constituyen las características que presenta la estructura productiva nacional. Los tres mayores productores - entre los que aparecen repetidamente Sinaloa, Jalisco, el estado de México y Chiapas- aumentaron su participación en la oferta nacional de 35\% en 1992 a $42.5 \%$ en 2005. En especial, la participación de Sinaloa ${ }^{2}$ pasó de $5.6 \%$ a $21.68 \%$ en el mismo periodo, hecho que

2 Jorge Fernández Menéndez (2007) afirma que el progreso inusitado de Sinaloa no sólo en la agricultura sino en otros rubros, se explica en parte por el lavado de dinero proveniente del narcotráfico. Los consumidores de autos de lujo en ese estado están a menudo relacio nados con ese giro. 
coloca a este estado en un lugar privilegiado, pero que volatiliza al mismo tiempo al mercado nacional, pues cualquier acontecimiento adverso, llámese huracanes, plagas, sequías, etcétera, pondría en peligro el abasto de maíz blanco para el mercado nacional. Así ocurrió a finales de 2006 y principios de 2007, cuando se exportó parte de la cosecha sinaloense a Japón. En un artículo publicado por John Moody y Verónica García León (5 de marzo de 2007) se relata la situación del productor sinaloense Arnoldo Godoy, quien cuenta con 800 hectáreas (has) de cultivo de maíz. Mediante 11 tractores y cinco trilladoras alcanza una productividad de 10 toneladas por hectárea y piensa incrementarla en $10 \%$ en los próximos años. El productor asegura que con ese rendimiento y a un precio de mil 900 pesos por tonelada obtiene tres mil pesos de utilidad por hectárea. Gracias al sistema de riego, el señor Godoy ha venido aumentando su productividad de manera constante durante los últimos 25 años. Para productores de la talla de Godoy la restricción en la posesión de tierra ha sido un obstáculo para sus planes de expansión. La Constitución marca un máximo de 100 hectáreas por productor y hasta 25 veces más para el caso de empresas. Se calcula que en la actualidad $96 \%$ de los productores tienen menos de 10 has y $85 \%$ menos de cinco. Ello dificulta competir con superficies de 500 has o más que poseen los productores estadounidenses. A partir de 1992, con la reforma del Artículo 27 Constitucional, se permite a los ejidatarios vender sus parcelas, pero hasta la fecha sólo se ha podido privatizar $1 \%$ de la superficie ejidal. La dificultad estriba en reunir a todos los ejidatarios para determinar si uno o dos pueden vender su parcela. En virtud de que muchos han emigrado es muy difícil comprar terrenos.

Los créditos agropecuarios se destinan a los productores más eficientes y los instrumentos de financiamiento se han venido modernizando y van desde el clásico bancario hasta las sociedades financieras de objeto limitado (Sofol) agropecuarias. En todo el país, el maíz cultivado con sistemas de riego aumentó de $21 \%$ a $47 \%$ entre 1994 y 2004; sólo un millón de hectáreas se siembra con riego, el resto, más de seis millones, es de temporal y arroja rendimientos de dos a tres toneladas por hectárea, comercializan poco y consumen la mayor parte de su cosecha.

La gráfica 6 nos muestra la evolución de los precios internacionales y nacionales del maíz, así como la diferencia entre ambos. Después de 1993 hay una caída de los precios internos que reduce la diferencia con los precios internacionales. A partir de 1995 vuelve a elevarse la diferencia hasta 1998, año en que comienza a descender. Ello causó preocupación porque los precios bajos conducían a una descapitalización de los productores al desalentar a los menos productivos (Rita Schwentesius et al., 2007). 
Gráfica 6

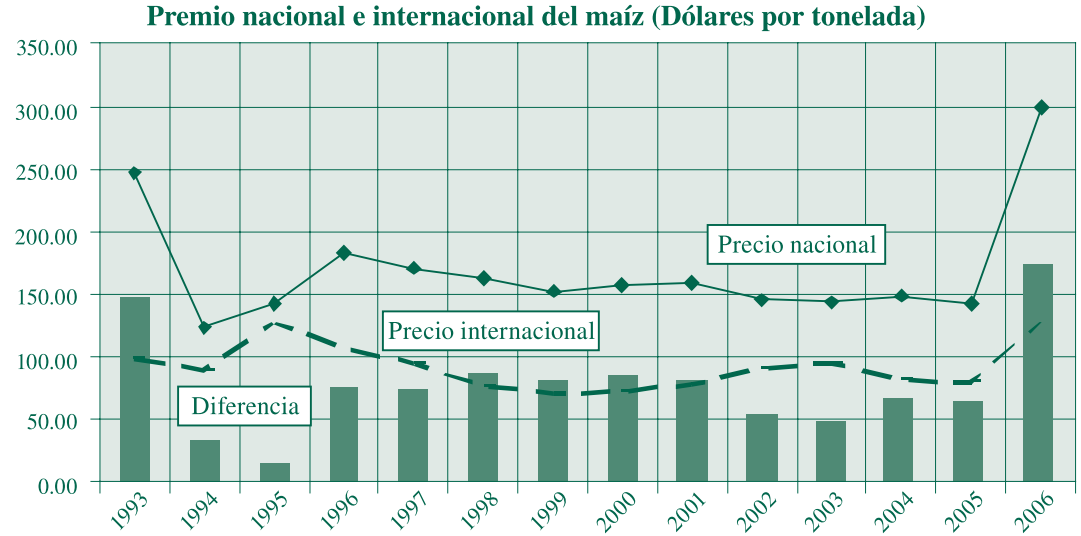

Fuente: SAGARPA (2007). Datos básicos del maíz y series históricas [http://www.siea.sagarpa.gob.mx /ar_comserhis.html].

Dentro del territorio nacional, los precios difieren de una entidad a otra y varía año con año. Con datos de la SAGARPA, se advierte de 1995 a 2005 una correlación entre las entidades que muestran mayor productividad —medida en toneladas por hectárea (t/has) y que osciló entre 2.8 y 8 - con los precios más bajos por tonelada. Si se seleccionan año con año los estados más productivos con los que ofrecieron precios más bajos por tonelada, existe en el periodo una coincidencia por orden de importancia en donde destacan Sinaloa, Sonora, Querétaro, Chihuahua, Jalisco, Baja California Sur, Guanajuato y Nayarit. Por otro lado, los estados con menor productividad (entre $0.7 \mathrm{t} /$ has y 1.5 t/has) y en donde el precio del maíz fue más alto fueron Oaxaca, Zacatecas, Coahuila, Quintana Roo y el Distrito Federal. Uno de los principales factores que elevan el precio interno del maíz es el costo del flete y el almacenamiento. La pobre estructura carretera y ferroviaria, así como la poca capacidad almacenadora explican en parte la diferencia en los precios. Por ejemplo, en 2005 la tonelada de maíz se vendió en Sinaloa a mil 301.59 pesos la tonelada, mientras que en Oaxaca se cotizó a un precio de dos mil 473.23 pesos, es decir, más de $80 \%$.

$\mathrm{El}$ horizonte favorece a los estados que concentran la mayor parte de la producción, especialmente Sinaloa y Jalisco. En este panorama, se espera una expansión del área de cultivo, un aumento de las importaciones de maquinaria agraria, un auge en las sociedades financieras de objeto limitado o múltiple, etcétera. No obstante, el gran negocio se perfila para empresas extranjeras como Monsanto, Dow, Du Pont, Cargill, Syngenta, entre otras. Las acciones bursátiles de las tres primeras se dispararon tras el anuncio del plan Bush relativo a las ambiciosas metas de producción de etanol. Según 
el Consejo Nacional Agropecuario, el promedio de productividad se podría duplicar con el uso de la biotecnología. Se afirma que la ingeniería genética tiene un gran potencial para producir maíz con alto porcentaje de almidón, insumo preponderante en la producción de etanol. Los productores de Sonora, Tamaulipas y Jalisco, por su parte, manifestaron ya su interés en la experimentación con transgénicos para elevar la productividad y así enfrentar la apertura comercial del grano que bajo el Tratado de Libre Comercio de América del Norte (TLCAN) tendrá lugar a partir de enero de 2008. En 2006 la siembra de cultivos genéticamente modificados sumó 102 millones de hectáreas en manos de 10.3 millones de agricultores de 22 países. Estados Unidos lleva el liderazgo con $25 \%$ de maíz, $57 \%$ de soya, $13 \%$ de algodón y $5 \%$ de canola ( $E l$ Financiero, 23 de 2007:15). Son 2.5 millones de productores que quedarán fuera del auge por venir. En un estudio hecho en comunidades de autoconsumo (Reyes, 2005) se concluyó que la mayoría de los pequeños productores de maíz enfrentan costos de producción muy superiores al precio de venta. Su actividad productiva se explica por factores antropológicos y sociales más que de rentabilidad.

\section{Repercusiones sociales}

Sin duda, uno de los principales problemas que se derivaron del aumento de los precios internacionales del maíz es que afectó a varios productos de la canasta básica, comenzando por la tortilla. A principios de enero de 2007, el kilogramo de este producto se llegó a vender hasta en 30 pesos en Durango y a 18 pesos en Baja California; en la ciudad de México y en la zona conurbada se mantuvo entre 10 y 15 pesos. ( $\mathrm{La}$ Jornada 11 de enero de 2007).

Como lo señala la gráfica 7, la variación en los precios en el kilogramo de tortilla en los distintos estados de la república pasó de $5.02 \%$ en enero de 2006 a $19.13 \%$ en enero de 2007. A partir de febrero de ese año, la variación desciende a $16.10 \%$ en virtud de la entrada en vigor del Acuerdo para Estabilizar el Precio de la Tortilla (AEPT). Dicho acuerdo fue anunciado desde Los Pinos el 18 de enero de 2007 para poner un alto a la volatilidad del mercado y garantizar el abasto de maíz, harina de maíz y tortilla a un precio justo para la población. Se emitió con carácter temporal con vigencia hasta el 30 de abril pero se extendió hasta agosto de 2007. De ahí emanan los siguientes compromisos: a) garantizar un precio de venta al público estable, b) combatir la especulación e incrementos injustificados, c) asegurar el abasto en lugares apartados mediante DICONSA, d) compromiso de vender el kilogramo de maíz a no más de 3.50 pesos por parte de agentes comerciales, e) compromiso por parte de las empresas suscritas a la Asociación Nacional de Tiendas de Autoservicio y Depar- 
Gráfica 7

Variación de los precios del kg de tortilla e INPC
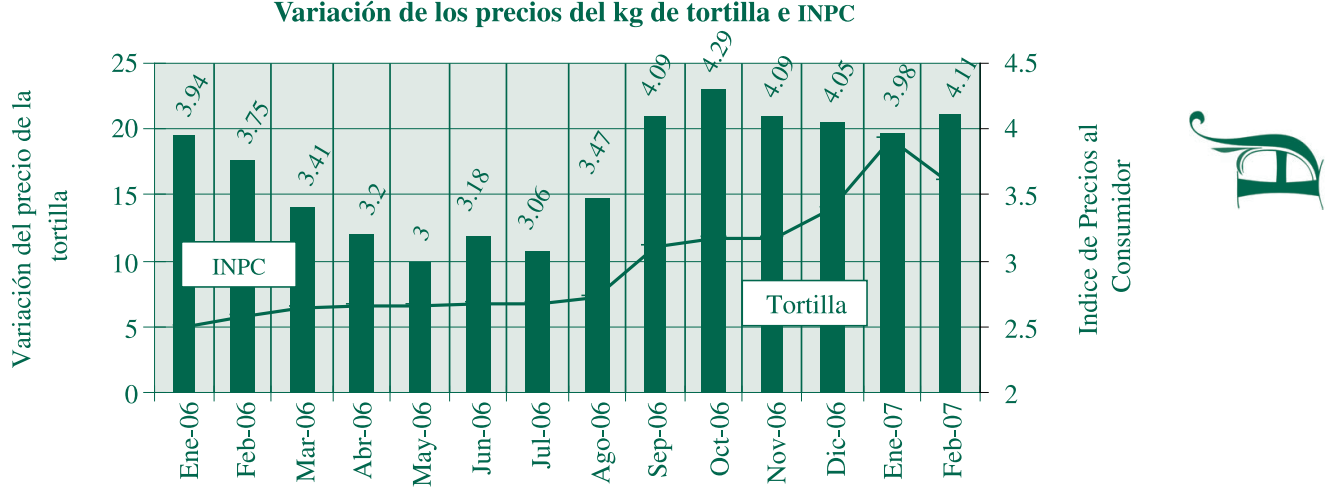

Fuente: El Financiero, 28 de febrero de 2007, p. 22); Banamex, 2007. Tasas de inflación [http://www.banamex.com/esp/finanzas/historicos/economia_int/ei_eua_cambio.jsp?init=2003\&offset=7]

tamentales y la cadena Wal Mart de vender el kilogramo de tortilla por debajo de los 6.00 pesos, f) incrementar del cupo de importación hasta 750 mil toneladas libre de arancel, así como dos millones 850 mil toneladas para atender la demanda del sector pecuario, g) alentar el incremento de la producción nacional y h) castigar los abusos con la intervención de la Procuraduría Federal del Consumidor y la Procuraduría General de la República.

A principios de 2007 se generó una intensa discusión en torno a quién o quiénes especulaban con el maíz. Empresas como Cargill, Maseca y Minsa fueron señaladas como responsables de la especulación por diversos medios, así como numerosos bodegueros con gran capacidad de almacenamiento. Araceli Damián (El Financiero, 22 de enero de 2007) señaló que el gobierno de Vicente Fox autorizó a Cargill comprar 600 mil toneladas de maíz a mil 650 pesos; ahora el precio que esa compañía fija para los molineros es de tres mil 500 pesos. Análogamente, se acusó a las grandes firmas harineras de alterar la calidad de la tortilla, mezclándola con harina de olote y así poder vender el kilo a precios concordantes con el AEPT. Al no existir una reglamentación y una metodología para identificar alteraciones en el producto, el consumidor no tiene forma alguna de objetar y percibir esas prácticas. Los miles de pequeños negocios que no pueden importar barato ni reducir costos no tienen otra opción que enfrentar la quiebra, ser denunciados ante las autoridades o dar precios al margen del AEPT como negocios no asociados. Se estima que existen 200 mil tortilleros en el ámbito nacional de los cuales sólo cinco mil se comprometieron con el AEPT.

Para junio de 2007 los precios del kilo de tortilla oscilaban en el territorio nacional entre 5.50 y 10.00 pesos. Sin embargo, el impacto sobre productos derivados, 
bienes de la canasta básica y el nivel general de precios fue mayor de lo que las autoridades esperaban. Como se aprecia en la gráfica 7, el Índice Nacional de Precios al Consumidor (INPC) acumulaba presiones desde finales de 2006 y más a partir de un incremento de $4.16 \%$ en el precio de la gasolina de alto octanaje, 3.3\% en el diesel y un peso en la leche DICONSA en noviembre de ese año. Si bien en enero de 2007 la inflación anual fue de 3.98, para febrero ya había aumentado a $4.11 \%$ por el efecto de la tortilla y el maíz.

Cuadro 7

Incremento en costos de producción y precios

\begin{tabular}{lcc}
\hline Producto & $\begin{array}{c}\text { Incremento (\%)en los } \\
\text { costos de producción } \\
\text { (ene 06-abr 07) }\end{array}$ & $\begin{array}{c}\text { Incremento } \\
\text { en los precios de (\%) } \\
\text { (ene 07-abr 07) }\end{array}$ \\
\hline Leche & 11.8 & 4.9 \\
Pollo & 19.9 & 10.4 \\
Huevo & 18.2 & 22.60 \\
Tortilla de maíz & 45.5 & 16.00 \\
Carne de cerdo & 21.2 & 1.98 \\
\hline
\end{tabular}

Fuente: El Financiero, 7 de mayo de 2007 y 10 de mayo de 2007.

El precio de maíz amarillo utilizado como forraje acumuló un incremento de 40.94\% de enero de 2006 a abril de 2007, mientras que el sorgo lo hizo en 39.28\%. Es decir, los granos forrajeros subieron un promedio de $40 \%$, lo que se tradujo en un aumento promedio de $14.3 \%$ de los costos de producción de las empresas que utilizan esos insumos en la elaboración de leche, huevo, carne de bovino y cerdo (véase el cuadro 7). Por otro lado, los precios a la venta no se ajustaron de manera proporcional, por lo que muchos productores sufrieron severos recortes en su tasa de utilidad y otros inclusive la quiebra. Este golpe al sector agropecuario traerá como consecuencia una caída en la producción nacional, el incremento de las importaciones y el desempleo en dimensiones hasta ahora poco estudiadas. Las presiones inflacionarias derivadas de este fenómeno fueron temporalmente neutralizadas por el Banco de México el 27 de abril de 2007 al subir la tasa de referencia en 1/4 de punto al pasar de $7 \%$ a $7.25 \%$, medida que esa institución calificó como preventiva. El gobernador del Banco de México ${ }^{3}$ confió en que el AEPT lograría sus objetivos y aseguró que la restricción monetaria no afectaría las tasas comerciales en virtud de que el ajuste tenía un carácter marginal.

3 Añadió que cerca de $60 \%$ de los créditos y $100 \%$ de los financiamientos personales se ha bían contratado con una tasa fija; en el caso de los créditos hipotecarios 65\% eran de tasa fija y $35 \%$ de tasa variable, pero con límites al alza. 
El aumento de los precios en la canasta básica empobrecerá aún más a las personas con bajos recursos. Los mayormente afectados son los 18 millones de personas que se encuentran en la llamada pobreza alimentaria o extrema. Son individuos cuyo ingreso mensual es menor de 790.74 en las zonas urbanas y 584.34 pesos en las zonas rurales. Se calcula que las familias en condición de pobreza aumentarán su gasto

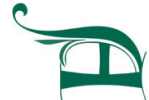
mensual en consumo de tortilla de 65.40 a 92.60 pesos, mientras que el incremento salarial para 2007 fue sólo de 4\%. En este contexto, el temor manifestado por las autoridades de detener para este año en lo posible toda demanda salarial superior a la cifra oficial del INPC — pues de lo contrario se desataría una espiral inflacionaria de precios y salarios- parece poco fundamentado. No es difícil suponer una participación de los salarios en el costo total del producto en la mayoría de las empresas medianas y grandes de casi $20 \%$ del producto, por lo que un aumento salarial de $5 \%$ sólo haría subir el precio final de las mercancías en 1\%; ello sin contar que el aumento de los precios no está siempre en relación directa con los costos, sino que intervienen de manera importante factores de competitividad y elasticidad de demanda de los productos.

Finalmente, es probable que se esté frente a un incremento generalizado de precios en granos a partir de transformaciones estructurales de consumo en el ámbito mundial y que, por tanto, las presiones internas al INPC continúen hasta 2008. ${ }^{4}$ Según el Bank of America (Sheridan, 2007:34), el PIB combinado de 24 grandes países emergentes se ha duplicado de 2002 a 2007, haciendo que el ingreso per capita se incremente en $14 \%$. Entre mayor es el ingreso, mayor es el consumo de carne y mayor la demanda de granos. En China, el consumo de carne de res y de puerco aumentó en $26 \%$ y $19 \%$ en cada caso desde 2000. Análogamente, el consumo de pollo en India se duplicó en siete años. Se calcula que por cada kilogramo de carne se requieren siete kilogramos de alimento.

\section{Conclusiones}

El cambio climático cobra cada vez mayor importancia por ser un problema global. Experimentar con el uso de nuevas fuentes de energía para disminuir la emisión de gases contaminantes es una prioridad. No obstante, el ambicioso proyecto de Estados Unidos amenaza con crear mayores problemas que soluciones. Cultivar maíz y azúcar en cantidades exorbitantes supone una amenaza a la seguridad ali-

4 Un aspecto de presión adicional lo constituye sin duda el impacto que tendrá en el INPC la reforma fiscal aprobada en el segundo semestre de 2007. 
mentaria y al mismo equilibrio ecológico, pues tal proceso va de la mano con la extensión de sistemas de riego que requieren grandes cantidades de agua, desplaza el cultivo de otros productos como ha ocurrido en Brasil y promueve el uso de semillas genéticamente modificadas, cuyo efecto, aunque controvertido, no deja de representar incertidumbre. Países como China e India requerirán cantidades crecientes de grano para alimento humano, por lo que se podría generar un aumento en los costos de producción en el ámbito internacional que tarde o temprano se manifestaría en mayor inflación. En síntesis, el proyecto de George W. Bush que se presenta como ecológico amenaza con crear escasez de alimento y de agua en los países productores y abrir paso a las firmas estadounidenses en Centroamérica y parte de Sudamérica, especialmente en lo que toca a corporaciones especializadas en tecnología genética.

Para México, y de no tomar medidas preventivas, este horizonte apunta hacia una polarización aún mayor del sector agrícola. Los grandes productores de maíz se asociarán con empresas de alta tecnología y se sumarán al proyecto del etanol en pro de la modernización y el progreso. Se agilizará la posibilidad de adquirir más extensiones de tierra mediante más reformas a la ley, lo que implicará la expulsión de más propietarios de sus pequeñas parcelas (Calva, J. L., 1993) poniendo en riesgo la seguridad alimentaria de la población de menos recursos. El uso de economías de escala y transgénicos acelerará, por un lado, la explotación y contaminación de mantos acuíferos y, por el otro, la depredación de la biodiversidad, principalmente en lo que toca a variedades de maíces criollos, cultivados en regiones pobres e indígenas. Ante un eventual aumento de los precios del maíz, la industria pecuaria perderá competitividad y se incrementarán las importaciones, lo que a su vez elevará el desempleo y el deterioro de la balanza comercial. El proyecto de George W. Bush traerá a México una mayor iniquidad en la distribución del ingreso, de no tomar ahora medidas que amortigüen el impacto.

En virtud del continuo agotamiento de reservas de petróleo y la falta de recursos para explorar y explotar nuevos pozos, no es posible ignorar la opción del etanol como biocombustible. Pero ello requiere de una cuidadosa estrategia de transición que privilegie, por ejemplo, los siguientes aspectos: a) alentar la participación de empresas nacionales en la producción de etanol, b) limitar el uso de la tierra para tal fin con el objeto de evitar la sobreexplotación de mantos acuíferos, c) evitar que la recomposición de cultivos ejerza un efecto de expulsión en la producción de alimentos, y d) proteger la biodiversidad, así como las comunidades indígenas que se rigen por la tradición y el autoconsumo. 


\section{Bibliografía}

ASERCA, "Situación Internacional y perspectivas del mercado de maíz", [http://www.infoaserca.gob.mx/analisis/sublistaanalisis. html], extraído el 5 de marzo de 2007.

BANAMEX, "Tasas de inflación", [http:// www.banamex.com/esp/finanzas/historicos/economia_int/ei_eua_cambio. jsp?init=2003\&offset=7] extraído el 22 de enero de 2007,

Brzezinski, Zbigniew, El gran tablero mundial. La supremacía estadounidense y sus imperativos geoestratégicos, México, Paidós, 1997.

, Out of control. Global Turmoil on the eve of the 21 st century, Nueva York, Touchstone, 1995.

Calva, José Luis, La disputa por la tierra. La reforma al artículo 27 y la nueva ley agraria, México, Fontamara, 1993.

Castaings, Teilley, "Así vamos... los salarios ¿son inflacionarios?", El Financiero, 8 de febrero de 2007, p. 23.

Damián, Araceli , "Maíz y tortillas", El Financiero, 22 de enero de 2007, p. 14.

Der Tagesspiegel, "Eine weitere Erdewärmung verhindern", 20 de septiembre de 2006, p. B2.

Dudenhöffer, Ferdinand, "Ökologie leicht gemacht. So schont die Autoindustrie das Klima”, Die Zeit, 22 de febrero de 2007, p. 22.

El Financiero, "Avanza experimentación de maíces transgénicos", 23 de febrero de 2007, p. 15.

"Continuarán las presiones inflacionarias, advierte el CEESP", 7 de mayo de 2007, p. 15.

"Distorsiona al mercado uso pecuario del maíz blanco", 26 de enero de 2007 , p. 19.

, "Mayor impulso a la producción de biocombustibles en el mundo; en cinco años aumentó $20 \%$ la obtención de etanol", 22 de enero de 2007, p. 9A

"Persisten desajustes en la cadena maíz tortilla", 28 de febrero de 2007, p. 22.

, "Vigente, la distorsión de precios: la deflación general contrasta con el elevado costo de básicos", 10 de mayo de 2007, p. $3 \mathrm{~A}$.

, "Déficit anual de $6 \%$ en la producción de maíz. Creciente demanda del sector pecuario, CEESP", 30 de abril de 2007, p. 57.
Fernández Menéndez, Jorge y Victor Ronquillo, De las maras a los zetas", México, Debolsillo, 2007.

Grefe, Christiane, "Mais für die Welt", Die Zeit, 19 de agosto 2004, pp. 11-14.

Huntington, Samuel, The clash of civilizations and the remaking of world order, Nueva York, Touchstone, 1997.

Knochenhauer, Guillermo, "A producir más maíz", El Financiero, 23 de febrero de 2007, p. 43.

La Jornada, "Caos en el precio de la tortilla; llegó a \$30 en Durango y \$18 en Baja California", 11 de enero de 2007, [http://jornada.unam.mx/2007/01/11/index.php?sectio $\mathrm{n}=$ economia\&article $=019 \mathrm{n} 1 \mathrm{eco}]$.

Moody, John y Verónica García León, "Un maíz dos mundos", Expansión, 19 de febrero-5 de marzo de 2007, pp. 62-67.

National Corn Growers Asociation, "World of corn production", [http://www.ncga.com/ WorldOfCorn/main/production1.asp], extraído el 2 de junio de 2007

Nitsch, Manfred y Jens Giesdorf, "Biotriebstoffe in Brasilien", Wirtschaftliche Reihe, Berlín, Frei Universität Berlin, núm. 12, 2005.

Ojeda Bustamante, José. "Maíz, etanol y México: del desabasto a la incongruencia", Política, Culiacán, núm. 12, año 2, 2007, pp. 46-50.

Presidencia de la República, "Palabras del secretario de Economía durante la firma del Acuerdo para la Estabilizar el Precio de la Tortilla", [http://www.presidencia.gob.mx/ prensa/discursos/?contenido $=28667]$, extraído18 de enero de 2007.

Reyes Guzmán, Gerardo, Comercialización del maíz criollo en Puebla, Tlaxcala e Hidalgo, México, Lupus Inquisidor, 2005.

SAGARPA, "Datos básicos del maíz y series históricas", [http://www.siea.sagarpa.gob.mx/ ar_comserhis.html], extraído el 12 de mayo de 2007.

Schwentesius, Rita, M. A. Gómez, J. L. Calva Tellez, L. Hernández Navarro, ¿El campo no aguanta más?, Texcoco, Universidad Autónoma Chapingo, PIA-CIESTAAM, 2007.

Sen Antara, Dev, "Die Hälfte de indischen Bevölkerung hat keinen Strom. Es ist nicht fair, wenn wir für dir Energiesunden der reichen bezahlen sollen", Die Zeit, 6 de junio de 2007, pp. 45-46. 
Sheridan, Barrett, "Blame it on Biofuels. Cornflake makers and socialist alike are pointing to green fuel for high food prices. Are they right?", Newsweek, 27 de agosto de 2007, p. 34.

The Economist, "The heat is on. Survey of climate change", 9 de septiembre de 2006, pp. 3-8. , "Fuel for Friendship. America and its neighbors discover a common interest", 3 de marzo de 2007, pp. 44-46.
"The greening of America. How America y likely to take over leaderishipo of the fight against climate change; and how it can get it right", 27 de enero de 2007, p. 9.

"Waking up and catching up. Belatedly and for many reasons, America is embracing environmentalism", 27 de enero de 2007, pp. 22-24. 